\title{
openheart Enhancing the diagnosis of fabry disease in cardiology with a targeted information: a before-after control- impact study
}

Anne-Louise Savary, ${ }^{1}$ Remy Morello, ${ }^{2}$ Carole Brasse-Lagnel, ${ }^{3}$ Paul Milliez, ${ }^{1}$ Soumeya Bekri, ${ }^{3}$ Fabien Labombarda ${ }^{1}$

To cite: Savary A-L, Morello R, Brasse-Lagnel C, et al. Enhancing the diagnosis of fabry disease in cardiology with a targeted information: a before-after controlimpact study. Open Heart 2017;4:e000567. doi:10.1136/ openhrt-2016-000567

Received 11 November 2016 Revised 12 January 2017 Accepted 17 January 2017

\section{CrossMark}

'Department of Cardiology, CHU de Caen, Caen, France ${ }^{2}$ Department of Biostatistics, Rouen University Hospital, CHU de Caen, Caen, France ${ }^{3}$ Department of Metabolic Biochemistry, CHU de Rouen, Rouen, France

Correspondence to Dr Fabien Labombarda; fabien. labombarda@gmail.com

\section{ABSTRACT}

Background Cardiac complications in Fabry disease are frequent and dominated by a high frequency of left ventricular hypertrophy; therefore, cardiologists may have an essential role in screening for this disease. Providing cardiologists with targeted information on Fabry disease would be valuable and could reduce both diagnostic and therapeutic delays. The aim of this study was to evaluate the efficiency of such strategy for Fabry screening. Methods We conducted a before-after control-impact study by comparing observations made before and after targeted information on Fabry disease among cardiologists. The information on Fabry disease consisted of (1) an educational booklet, (2) oral information and (3) screening kits. The programme was evaluated at the end of a 12-month study period.

Results Forty-two cardiologists participated to this study. None of them had conducted screening test and new diagnostic for Fabry disease in the 3 years prior the information. After the information, screening with dried blood spots was performed in 55 patients (ranged 18-77 years, men: 39) with cardiac monitoring for supposed sarcomeric hypertrophic cardiomyopathy $(n=41)$ or unexplained left ventricular hypertrophy $(n=14)$ from January 2015 to January 2016. Two new cases of Fabry disease were diagnosed (3.4\%) in two men (ages 58 and 51 years). The information was deemed relevant in both content and structure and was deemed useful for everyday practice.

Conclusion Cardiologists valued the targeted information on Fabry disease. This information had a direct clinical impact by allowing the diagnosis of two new families with Fabry disease.

\section{INTRODUCTION}

Fabry disease is an inherited metabolic disease related to a deficiency of $\alpha$-galactosidase A. This enzyme is responsible for a progressive accumulation of glycosphingolipids, such as globotriaosylceramide (Gb3), in the lysosomes of many cell types. ${ }^{1}$ Fabry disease diagnosis is often delayed and occurs after the onset of severe clinical signs, which include cardiac symptoms. ${ }^{2}$ Cardiac complications in Fabry disease are present in more

\section{KEY MESSAGES}

What is already known about this subject?

- Cardiac complications in Fabry disease, especially left ventricular hypertrophy, are frequent and represent now the leading cause of death in patients with Fabry disease. Therefore, cardiologists may have an essential role in the screening of this rare metabolic disorder. The recent guidelines on Hypertrophic Cardiomyopathy of the European Society of Cardiology have strengthened their message regarding the importance of investigating rare causes of left ventricular hypertrophy, such as Fabry disease. However, screening for Fabry disease remains suboptimal, which may result in 'loss of opportunity' for these patients, with diagnostic and therapeutic delay. The importance of the early diagnosis of cardiac involvement is well established in Fabry disease since specific therapy is more effective when instituted prior to the onset of irreversible damage, such as fibrosis.

What does this study add?

- In our study, we demonstrated that a targeted information on Fabry disease, provided over a short period of time among general cardiologist, had a direct clinical impact by allowing the diagnosis of two new families with Fabry disease. Cardiologists reported that the information on rare disease such as Fabry disease was useful for their everyday practice.

How might this impact on clinical practice?

- A targeted information on Fabry disease among cardiologists may be a simple way to improve the screening and reduced both diagnosis and therapeutic delay of Fabry disease.

than half of patients and are dominated by a high frequency of left ventricular hypertrophy $(\mathrm{LVH}){ }^{3}$ Cardiologists are the first-line healthcare providers to diagnose $\mathrm{LVH}$ via ECG, echocardiogram or resonance magnetic nuclear (RMN) imaging. Therefore, they have an essential role in both the screening 
and diagnosis of Fabry disease. As shown in other rare disorders, a diagnostic delay in patients with Fabry is unfavourable and may cause a 'loss of opportunity' for patients by delaying optimal medical management. ${ }^{4} \mathrm{~A}$ lack of information on this rare condition in the medical community including the cardiologists may explain the delay in diagnosis. ${ }^{5}$ Targeted educational interventions for health professionals can effectively improve the screening and diagnosis of rare diseases. Additionally, specific therapy is more effective when instituted early ${ }^{6}$ in patients with Fabry disease. Thus, targeted interventions for the early screening of Fabry disease may be relevant and could reduce delays in diagnosis and therapeutic interventions. The aim of our study was to determine whether providing targeted information to cardiologists improves the screening and diagnosis of Fabry disease.

\section{METHODS}

We conducted a before-after control-impact study among cardiologists of our region (Normandy, France, population area: 1.5 million) by comparing observations made before and after a targeted information on Fabry disease. This information consisted of the following: (1) an educational booklet, (2) oral information and (3) screening tools. During the information, a short questionnaire was given to each participant to know how many patients were screened and diagnosed for Fabry disease during the 3 years prior the information. After a 12-month study period, participating cardiologists were contacted to evaluate the targeted information on Fabry disease and to collect retrospectively the clinical data of the screened population. This study was approved by our local ethics committee (CPP Nord-Ouest III).

\section{Educational booklet and oral information}

An educational booklet (online supplement file) was developed by the Caen University Hospital for Hereditary Cardiomyopathies (Caen-CHC). The oral information consisted of a $10 \mathrm{~min}$ presentation by a cardiologist working at the Caen-CHC. The presentation addressed the content of the booklet and described the candidates for the screening. Based on experiences in our centre and the recent hypertrophic cardiomyopathy (HCM) guidelines of the European Society of Cardiology, ${ }^{7}$ candidates for Fabry disease screening should meet the following criteria: patients $>18$ years, LVH of unknown aetiology defined by a wall thickness $\geq 15 \mathrm{~mm}$ measured on echocardiography or RMN, absence of father-son transmission, absence of known family Fabry disease and signed consent for genetic analysis.

\section{Kits for biochemical screening and genetic analysis}

The Fabry disease screening kits included a filter paper and a protocol for the collection of dried blood. The measurement of $\alpha$-galactosidase A activity and molecular studies were centralised (Metabolic Biochemistry Department, Rouen University Hospital, Normandy, France). Enzyme activities were measured using a tandem mass-based multiplex assay. ${ }^{8}$ Men with low $\alpha$-galactosidase A activity and all women were subjected to genetic analysis of the GLA gene. Genomic DNA was extracted from venous blood using QIAamp DNA Blood Mini Kit Qiagen and was amplified in vitro by PCR. Multiple pairs of primers were synthesised to amplify each of GLA exonic regions. Primers used to amplify the genomic sequences were designed according to the sequence NM_000169.2 5. Direct DNA fragments sequencing was performed with an ABI prism big dye Terminator cycle Sequencing Ready Reaction Kit (PE Applied Biosystems/and ABI model 3130xl Genetic Analyzer). Patients' genomic sequences comparison with the reference sequence is done by Variant Reporter software (Applied Biosystems). The identified variations are mined by ALAMUT software (Interactive-Biosoftware). The described variations are named following the current nomenclature recommendations (http://www.hgvs.org/mutnomen).

\section{RESULTS}

A total of 42 cardiologists received the targeted information on Fabry disease. The results showed that 25 (60\%) had exclusive hospital activity, $14(33 \%)$ had a combination of liberal and hospital joint activity and $3(7 \%)$ had an exclusive liberal practice. There were no physicians who had conducted screening and new diagnostic for Fabry disease in the 3 years prior to being provided the targeted information. Ninety-three per cent of cardiologists thought more education on Fabry disease was needed in their medical training, 93\% found the targeted information appropriate in both content and structure and $86 \%$ found it useful for their daily practice.

\section{Screened population}

The cardiologists conducted filter paper testing on 55 patients (age: $54 \pm 15$ (18-77 years), men: 39) from January 2015 to January 2016. The testing was performed in patients with supposed sarcomeric HCM $(n=41)$ or in patients with LVH due to unknown aetiology and/or considered as disproportionate compared with treated and well-controlled hypertension $(n=14)$. The data show $54 \%$ of patients had a history of hypertension and $20 \%$ had a history of stroke. The most frequent symptoms were dyspnoea, angina and supraventricular arrhythmia. Patient characteristics are shown in table 1.

\section{Patients with Fabry disease}

The screening allowed the identification of Fabry disease in two male patients. The characteristics of the patients with Fabry are reported in table 2. The first patient was a 58-year old man and had been followed for more than 5 years for HCM with an asymmetric and diffuse LVH (figure 1A). This patient presented with a severe aortic valve regurgitation that led to an aortic valve replacement associated with a multiple coronary artery bypass graft. The second patient was 51 years old and had been followed for 15 years due to symptomatic obstructive 
Heart failure and cardiomyopathies

\begin{tabular}{|c|c|}
\hline Variables & \\
\hline \multicolumn{2}{|l|}{ Demographics } \\
\hline Age & $54 \pm 15(18-77)$ \\
\hline Sex & $39(71 \%)$ \\
\hline \multicolumn{2}{|l|}{ Familial history } \\
\hline Family history of sudden death & $10(18 \%)$ \\
\hline Family history of HCM & $7(12 \%)$ \\
\hline \multicolumn{2}{|l|}{ Medical history } \\
\hline Hypertension & $30(54 \%)$ \\
\hline Diabetes & $6(11 \%)$ \\
\hline Obesity & $16(29 \%)$ \\
\hline Dyslipidemia & $25(45 \%)$ \\
\hline Stroke & $11(20 \%)$ \\
\hline Chronic renal failure* & $8(14 \%)$ \\
\hline \multicolumn{2}{|l|}{ Pacemaker } \\
\hline \multicolumn{2}{|l|}{ Symptoms } \\
\hline NYHA I & $38(69 \%)$ \\
\hline$\|$ & $13(23 \%)$ \\
\hline III & $4(7 \%)$ \\
\hline IV & 0 \\
\hline Syncope & $6(11 \%)$ \\
\hline Angor & $8(14 \%)$ \\
\hline Atrial arrhythmia & $12(22 \%)$ \\
\hline Ventricular arrhythmia & $7(13 \%)$ \\
\hline No symptoms & $16(29 \%)$ \\
\hline \multicolumn{2}{|l|}{$E C G$} \\
\hline Sinusal rhythm & 37 (84\%) \\
\hline Short PR interval† & $1(1.5 \%)$ \\
\hline Sokolow (mm) & $27.5 \pm 13$ \\
\hline \multicolumn{2}{|l|}{ Echocardiography } \\
\hline Maximal wall thickness & $19 \pm 5(14-30)$ \\
\hline LVEF & $67 \pm 12$ \\
\hline LVOTO & $6(11 \%)$ \\
\hline Binary endocardium & $1(1.5 \%)$ \\
\hline
\end{tabular}

Table 2 Characteristics of patient with hemizygous Fabry disease

\begin{tabular}{lll}
\hline Characteristics & Patient 1 & Patient 2 \\
\hline Demographics & & \\
Age & 58 & 51 \\
Sex & $\mathrm{m}$ & $\mathrm{m}$
\end{tabular}

$\alpha \mathrm{Gal}$ activity and genetic

$\begin{array}{lll}\alpha \text { Gal }(\mu \mathrm{mol} / \mathrm{L} / \text { hour })^{*} & 0.7 & 1.6 \\ \text { Lyso Gb3 }(\mathrm{ng} / \mathrm{mL}) \dagger & 9.8 & 1.2 \\ \text { Mutation } & \text { c.644A>G p. N215S } & \text { Arg118Cys }\end{array}$

Medical history and symptoms

\begin{tabular}{lll}
\hline Familial history of HCM & 0 & 0 \\
\hline Hypertension & 0 & 1 \\
\hline Stroke & 0 & 0 \\
\hline Chronic renal failure & 0 & 0 \\
\hline Angiokeratoma & 0 & 0 \\
\hline Acroparesthesia & 0 & 0 \\
\hline Cornea opacities & 0 & 0 \\
\hline Angor & 1 & 0 \\
\hline Arrhythmia & 0 & 0 \\
\hline Pacemaker & 0 & 0 \\
\hline Echocardiography & & \\
\hline Wall thickness & 22 & 21 \\
\hline Binary endocardium & 0 & 0 \\
\hline LVOTO & 0 & 1 \\
\hline Valvulopathy & AR & RMN \\
\hline RV Hypertrophy & 0 & 0 \\
\hline RMN & & 850 \\
\hline T1 mapping (ms) & 730 & Absent \\
\hline LGE fibrosis & Absent & \\
\hline
\end{tabular}

*Reference value $\geq 1.8 \mu \mathrm{mol} / \mathrm{L} / \mathrm{hour}$.

†Reference value $\leq 1.8 \mathrm{ng} / \mathrm{mL}$.

$\alpha$ Gal, $\alpha$ galactosidase A activity; HCM, hypertrophic cardiomyopathy; LGE, late gadolinium enhancement; LVOTO, left ventricular outflow tract obstruction; Lyso Gb3, Lyso globotriaosylceramide; RMN, resonance magnetic nuclear; RV, right ventricular.

*Defined by a clearance of the creatinine $<60 \mathrm{~mL} / \mathrm{min}$.

†Defined by an PR interval $<120 \mathrm{~ms}$.

HCM, hypertrophic cardiomyopathy; PR, intervalle Pwave-Rwave.

HCM (figure 1B), which was treated by myomectomy and mitral valve replacement. The patient had no significant coronary artery disease. The systolic ventricular function was normal in both patients and there was no right ventricular hypertrophy or aortic dilatation observed. These patients did not require a pacemaker and they have no apparent family history of Fabry disease. The patients had no extra cardiac signs of Fabry disease; thus, there was no proteinuria, cornea abnormalities or sequelae of stroke noted by MRI. Patient 1 had abnormally low 


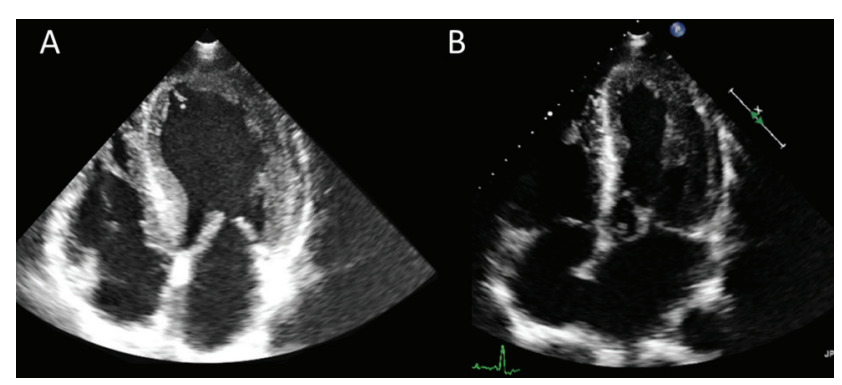

Figure 1 Hypertrophic cardiomyopathy (transthoracic echocardiogram). (A) Asymmetric diffuse LVH; (B) moderate and asymmetric $\mathrm{LVH}$ with systolic anterior motion of the mitral valve. LVH, left ventricular hypertrophy.

patient's refusal while this testing allowed the identification of a heterozygous relative of patient 2 without phenotypic expression.

\section{DISCUSSION}

Our data indicate that cardiologists valued the targeted information on a rare disease such as Fabry disease. The information was deemed relevant in both content and structure. Additionally, the physicians reported that the information was useful for their everyday practice. This targeted information had a direct clinical impact and led to the diagnosis of two new patients with Fabry. A targeted information among cardiologists may be a simple way to improve the screening and reduced the diagnosis delay of a rare metabolic disorder such as Fabry disease.

\section{How to improve the screening of a rare disease such as Fabry disease in cardiology? \\ Targeted information}

With an estimated prevalence between 1/40 000 and $1 / 117000$, Fabry disease is part of the so-called orphan diseases group. It shares several common features with this large and heterogeneous group of diseases, resulting in the difficulty of diagnosis, complexity of their organ damage, chronic evolution and the need for a complex multidisciplinary approach. Fabry disease is often diagnosed late, after the first signs of disease, and the average delay is $>10$ years between symptom onset and diagnosis. ${ }^{59}$ Cardiac involvement is responsible for its high morbidity and mortality and can reduce the life expectancy of men by 15 to 20 years. ${ }^{10}$ Its cardiac complications are currently the leading cause of death in patients with Fabry disease. ${ }^{11}$ The importance of the early diagnosis of cardiac involvement is well established in Fabry disease since specific therapy is more effective when instituted prior to the onset of irreversible damage, such as fibrosis. ${ }^{69}$ Similar to other orphan diseases, a diagnostic delay in Fabry disease may result in 'loss of opportunity' for the patients. ${ }^{10}$ Networks were developed in Europe and North America to improve the overall care of patients with rare disease. These networks involve cooperation between governments, research institutes, patient associations and the pharmaceutical industry. Since 2004, there have been two national plans for rare diseases in France. The objectives of these plans are to improve the identification and referral of patients with rare diseases, reduce the diagnostic delay, adapt patient management and improve communication between professionals. ${ }^{12}$ One important aspect of these plans focuses on screening and medical education. Fabry disease has benefited from this national and international group for rare diseases. Simple screening tools, such as Dried Blood Screening test (DBS), have been developed and made available for physicians. ${ }^{13}$ The recent guidelines on HCM of the European Society of Cardiology have strengthened their message regarding the importance of investigating rare causes of $\mathrm{LVH}$, such as Fabry disease. ${ }^{714}$ However, screening for Fabry disease in our region remains suboptimal. The use of the screening test was very low in the 3 years prior to our work and contrasts with the high frequency of LVH in the population. The two patients with Fabry diagnosed in our study had received cardiac monitoring for more than 5 years. This result illustrates the persistent delay in the diagnosis of Fabry disease and highlights the limitations of the educational programmes on rare diseases. Our educational intervention was designed to overcome these limitations by providing cardiologists with targeted information on Fabry disease. Although this information is readily available on the internet, it seems to have little impact in the medical field due to the lack of trust in the online information. ${ }^{15}$ Previous studies of physician education programmes on rare diseases have shown traditional teaching by a clinical expert remains a favourite mode of intervention for physicians. ${ }^{16}$ Our results are consistent with these data.

\section{A physician-friendly screening tool}

The use of DBS was proposed as a Fabry disease screening method instead of the measurement of $\alpha$-galactosidase A activity on leucocytes. ${ }^{17}$ Previous studies confirmed the reliability of this test, and its sensitivity and specificity are $100 \%$ in men. ${ }^{18}$ Although its sensitivity is lower in female patients (approximately 66\%), this test remains useful and can diagnose the disease in cases with reduced enzyme activity. ${ }^{19}$ The use of filter paper is simple, and the test is well accepted by patients in our experience. Additionally, sample transport and conservation are simple. These features make the DBS an ideal screening tool that is easy to use, affordable and can be used at the time of consultation.

\section{Cardiologists: key players for the diagnosis of Fabry disease} Heart involvement, and particularly LVH, is important in the diagnosis of Fabry disease before resultant renal ${ }^{20}$ or neurological ${ }^{21}$ damage. The cardiologist has an essential role in screening identifying LVH via ECG, echocardiography or MRI. Thus, cardiologists are key players and must be properly informed to reduce the delay in diagnosis and initiation of enzyme replacement therapy. Physician education should focus on two major questions: Which patients must be screened? What is the regional 


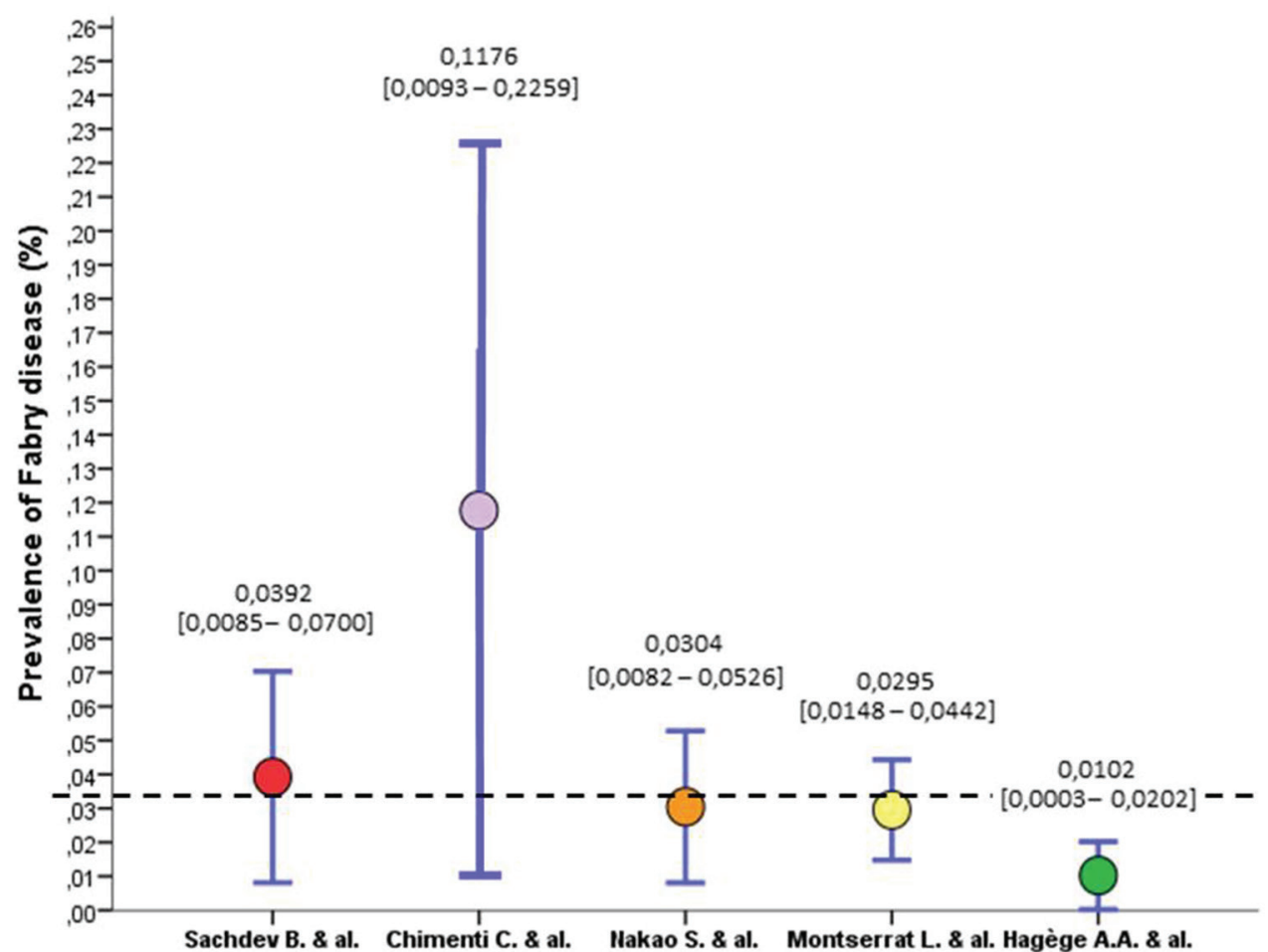

Figure 2 Prevalence of Fabry disease in hypertrophic cardiomyopathy populations. Dotted line represents the frequency of Fabry disease in our population.

addressing network in the case of suspected or confirmed Fabry disease? This educational role is part of the mission of the Centre for Hereditary Cardiomyopathies.

\section{Prevalence of Fabry disease in a population of HCM}

In previous studies with systematic searches for Fabry disease in patients with apparently unexplained $\mathrm{LVH}$, the prevalence of Fabry disease varied between $1 \%$ and $12 \%{ }^{22}$ (figure 2, see online supplement file). The differences between the populations screened and the screening methods used to measure $\alpha$-galactosidase A activity may explain the different prevalence reported by these studies. Although our study was not designed to assess the prevalence of Fabry disease in patients with $\mathrm{LVH}$, our data on a small population are consistent with the literature..$^{23} 24$ However, the phenotype of our two patients was unusual, given the reported low frequency of obstructive cardiomyopathy and surgical aortic regurgitation in Fabry disease. ${ }^{25}$

\section{Limitations}

Cardiologists who accepted to receive the targeted information were the most motivated, which may represent a sample bias. Our study was conducted over a limited period. Therefore, we cannot evaluate if the effects of the information will persist in a medium and a long term with a stable screening rate.

\section{CONCLUSION}

The cardiologist is a key player in the screening and diagnosis of Fabry disease because of the high frequency of cardiac involvement and LVH. Targeted information on
Fabry disease, provided over a short period of time, had a direct clinical impact by allowing the diagnosis of two new families with Fabry disease. A targeted information on Fabry disease among cardiologists may be a simple way to improve the screening and reduced both diagnosis and therapeutic delay of a rare metabolic disorder such as Fabry disease.

Acknowledgements The authors thank SHIRE France and the Association pour la Promotion de la Cardiologie et de la Recherche en Basse Normandie (APCRBN) for their assistance in the production of this work.

Contributors FL and RM: concept/design; ALS, FL and RM: data analysis/ interpretation; ALS and FL: drafting article; PM, SB, RM and CBL: critical revision of article; ALS, RM, CBL, SB, PM and FL: approval of article. All available data can be obtained bycontacting the corresponding author.

Competing interests None declared.

Ethics approval This study was approved by our local ethics committee (CPP Nord-Ouest III), a patient consent statement was obtained.

Provenance and peer review Not commissioned; externally peer reviewed.

Open Access This is an Open Access article distributed in accordance with the Creative Commons Attribution Non Commercial (CC BY-NC 4.0) license, which permits others to distribute, remix, adapt, build upon this work non-commercially, and license their derivative works on different terms, provided the original work is properly cited and the use is non-commercial. See: http://creativecommons.org/ licenses/by-nc/4.0/

(c) Article author(s) (or their employer(s) unless otherwise stated in the text of the article) 2017. All rights reserved. No commercial use is permitted unless otherwise expressly granted.

\section{REFERENCES}

1. Zarate YA, Hopkin RJ. Fabry's disease. Lancet 2008;372:1427-35.

2. Linhart A, Lubanda JC, Palecek T, et al. Cardiac manifestations in fabry disease. J Inherit Metab Dis 2001;24:75-83. 
3. Yousef Z, Elliott PM, Cecchi F, et al. Left ventricular hypertrophy in fabry disease: a practical approach to diagnosis. Eur Heart $J$ 2013;34:802-8.

4. Weidemann F, Linhart A, Monserrat L, et al. Cardiac challenges in patients with fabry disease. Int J Cardiol 2010;141:3-10.

5. Thomas AS, Mehta AB. Difficulties and barriers in diagnosing fabry disease: what can be learnt from the literature? Expert Opin Med Diagn 2013;7:589-99.

6. Beer M, Weidemann F, Breunig F, et al. Impact of enzyme replacement therapy on cardiac morphology and function and late enhancement in fabry's cardiomyopathy. Am J Cardiol 2006:97:1515-8

7. Elliott PM, Anastasakis A, Borger MA, et al; Authors/Task Force members. 2014 ESC guidelines on diagnosis and management of hypertrophic cardiomyopathy: the task force for the diagnosis and management of hypertrophic cardiomyopathy of the european society of cardiology (ESC). Eur Heart $J$ 2014;35:2733-79.

8. Elliott S, Buroker N, Cournoyer JJ, et al. Pilot study of newborn screening for six lysosomal storage diseases using tandem mass spectrometry. Mol Genet Metab 2016;118:304-9.

9. Hagège A. Cardiac manifestations of Anderson-Fabry disease and efficacy of enzyme replacement therapy. Rev Med Interne 2010;31:S238-42.

10. Clarke JT. Narrative review: Fabry disease. Ann Intern Med 2007;146:425-33.

11. Mehta A, Clarke JT, Giugliani R, et al; FOS Investigators. Natural course of fabry disease: changing pattern of causes of death in FOS - Fabry Outcome Survey. J Med Genet 2009;46:548-52.

12. Guillevin $L$. The national plan for orphan rare diseases: nearly 10 years on. Rev Neurol 2013;169:S9-11.

13. Lukacs Z, Hartung R, Beck M, et al. Direct comparison of enzyme measurements from dried blood and leukocytes from male and female fabry disease patients. J Inherit Metab Dis 2007;30:614
14. Rapezzi C, Arbustini E, Caforio AL, et al. Diagnostic work-up in cardiomyopathies: bridging the gap between clinical phenotypes and ESC guidelines. Eur Heart J 2013;34:1448-58.

15. Cullen RJ. In search of evidence: family practitioners' use of the Internet for clinical information. J Med Libr Assoc 2002;90:370-9.

16. Zack P, Devile C, Clark C, et al. Understanding the information needs of general practitioners managing a rare genetic disorder (osteogenesis imperfecta). Community Genet 2006;9:260-7.

17. Caudron E, Moliere D, Zhou JY, et al. Recent advances of fabry disease screening for at risk population. Med Sci 2005;21:e48-50.

18. Lukacs Z, Hartung R, Beck M, et al. Direct comparison of enzyme measurements from dried blood and leukocytes from male and female fabry disease patients. J Inherit Metab Dis 2007;30:614.

19. Linthorst GE, Bouwman MG, Wijburg FA, et al. Screening for fabry disease in high-risk populations: a systematic review. J Med Genet 2010;47:217-22.

20. Saito O, Kusano E, Akimoto T, et al. Prevalence of fabry disease in dialysis patients: Japan fabry disease screening study (J-FAST). Clin Exp Nephrol 2016;20:284-93.

21. Rolfs A, Fazekas F, Grittner U, et al; Stroke in Young Fabry Patients (sifap) Investigators. Acute cerebrovascular disease in the young: the stroke in young fabry patients study. Stroke 2013;44:340-9.

22. Hagège AA, Caudron $E$, Damy $T$, et al; FOCUS study investigators. Screening patients with hypertrophic cardiomyopathy for fabry disease using a filter-paper test: the FOCUS study. Heart 2011:97:131-6.

23. Nakao S, Takenaka T, Maeda M, et al. An atypical variant of fabry's disease in men with left ventricular hypertrophy. $N$ Engl J Med 1995;333:288-93.

24. Monserrat L, Gimeno-Blanes JR, Marín F, et al. Prevalence of fabry disease in a cohort of 508 unrelated patients with hypertrophic cardiomyopathy. J Am Coll Cardiol 2007;50:2399-403.

25. Weidemann F, Strotmann JM, Niemann M, et al. Heart valve involvement in fabry cardiomyopathy. Ultrasound Med Biol 2009;35:730-5 